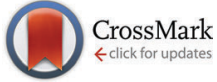

Cite this: Phys. Chem. Chem. Phys., 2015, 17, 30125

Received 13th August 2015 Accepted 14th October 2015

DOI: $10.1039 / c 5 c p 04819 b$

www.rsc.org/pccp

\title{
Charge transfer state emission dynamics in blue-emitting functionalized silicon nanocrystals $\dagger$
}

\author{
Glenda B. De los Reyes, ${ }^{\text {ab }}$ Mita Dasog, ${ }^{\text {cd }}$ MengXing Na, ${ }^{a}$ Lyubov V. Titova, ${ }^{\text {ae }}$ \\ Jonathan G. C. Veinot ${ }^{* C}$ and Frank A. Hegmann*a
}

\begin{abstract}
We explore the dynamics of blue emission from dodecylamine and ammonia functionalized silicon nanocrystals (Si NCs) with average diameters of $\sim 3$ and $\sim 6 \mathrm{~nm}$ using time-resolved photoluminescence (TRPL) spectroscopy. The Si NCs exhibit nanosecond PL decay dynamics that is independent of NC size and uniform across the emission spectrum. The TRPL measurements reveal complete quenching of core state emission by a charge transfer state that is responsible for the blue PL with a radiative recombination rate of $\sim 5 \times 10^{7} \mathrm{~s}^{-1}$. A detailed picture of the charge transfer state emission dynamics in these functionalized Si NCs is proposed.
\end{abstract}

\section{Introduction}

Nanostructured silicon $(\mathrm{Si})$ has received significant attention owing to its strong tunable visible photoluminescence (PL) that has potential applications in optical devices such as lightemitting diodes ${ }^{1,2}$ and silicon-based full color displays, ${ }^{3,4}$ as well as luminescent labels for biological imaging, ${ }^{5}$ among others. PL from silicon nanocrystals (Si NCs) can be tuned from infrared to visible wavelengths either by making the NCs smaller than the exciton Bohr radius $(\sim 4.5 \mathrm{~nm})^{6-9}$ or by designing the surface chemistry using organic compounds. ${ }^{10,11}$ While it is possible to achieve blue emission from quantum confinement (QC) in Si NCs with diameters less than $2 \mathrm{~nm}$, such small nanocrystals typically exhibit a significant reduction in the PL quantum yield (QY) due to higher nonradiative recombination rates. ${ }^{12,13}$ In the case of organic capped Si NCs, emission depends mostly on surface passivation. ${ }^{10,11}$ Compared to hydrogen-terminated Si NCs which exhibit QC emission, organic capped Si NCs show higher QY, better control of surface oxidation, more stable optical properties, and faster

\footnotetext{
${ }^{a}$ Department of Physics, University of Alberta, Edmonton, Alberta T6G 2E1, Canada.E-mail: delosrey@ualberta.ca, hegmann@ualberta.ca

${ }^{b}$ Department of Mathematics and Physics, University of Santo Tomas, Espana, Manila 1015, Philippines

${ }^{c}$ Department of Chemistry, University of Alberta, Edmonton, Alberta T6G 2G2, Canada. E-mail: jveinot@ualberta.ca

${ }^{d}$ Department of Chemistry and Chemical Engineering, California Institute of Technology, Pasadena, CA, 91125, USA

${ }^{e}$ Department of Physics, Worcester Polytechnic Institute, Worcester, Massachusetts 01609, USA

$\dagger$ Electronic supplementary information (ESI) available: Experimental details, material characterization, selected area diffraction pattern, TEM of $\sim 6 \mathrm{~nm}$ samples, normalized fluence-dependent TIPL, filter transmission spectrum, summary of the fitting parameters for spectrally-resolved PL dynamics, microsecond-TRPL measurements, and size-dependent absorbance. See DOI: 10.1039/c5cp04819b
}

recombination rates (on the order of $10^{7} \mathrm{~s}^{-1}$ ), which makes this material well suited for optoelectronics applications. ${ }^{11,14}$

Of the visible-range PL from Si NCs, blue emission is considered the most intriguing because of the relatively small indirect bandgap of bulk $\mathrm{Si}(1.1 \mathrm{eV})$ and the wide variety of potential emission mechanisms that have been proposed. Besides QC in Si NCs with diameters $d<2 \mathrm{~nm},{ }^{15,16}$ blue emission has also been discussed in terms of surface oxygen-related defects, ${ }^{17}$ amorphous surface layers, ${ }^{18}$ and near-interface traps. ${ }^{19}$ Recently, blue emission from charge transfer (CT) states in Si NCs has been suggested by Dasog et al. based on solvatochromic studies that showed a dependence of the PL peak emission on the polarity of the solvent. ${ }^{10,20}$ To date, a detailed study of the PL dynamics of these materials, which provides insights into the specific nature of the blue emission process, has not been performed.

In this letter, we used time-resolved photoluminescence (TRPL) to probe the emission dynamics in blue-emitting functionalized $\mathrm{Si}$ NCs with different NC sizes and surface passivation prepared by reacting dodecylamine with chloride-terminated $\mathrm{Si}$ NCs and ammonium bromide with hydride-terminated Si NCs. We show that the blue emission originates from a CT state at the $\mathrm{Si} / \mathrm{SiO}_{x} \mathrm{~N}_{y}$ interface. Understanding the dynamics of the CT emission process will have an impact on potential applications of such blue-emitting $\mathrm{Si}$ NCs in optoelectronics. In particular, since CT is an intermediate step for charge separation, it is important to understand CT dynamics for designing efficient Si NC-based solar cells.

\section{Materials and methods}

\subsection{Synthesis of functionalized Si NCs}

The syntheses of the colloidal Si NCs used in this study has been described in detail elsewhere. ${ }^{13,20,21}$ Briefly, commercial 
hydrogen silsesquioxane was thermally processed in a slightly reducing atmosphere $\left(95 \% \mathrm{Ar} / 5 \% \mathrm{H}_{2}\right)$ at $1100{ }^{\circ} \mathrm{C}$ (for $\left.\sim 3 \mathrm{~nm} \mathrm{Si} \mathrm{NCs}\right)$ and $1200{ }^{\circ} \mathrm{C}$ (for $\sim 6 \mathrm{~nm} \mathrm{Si} \mathrm{NCs)} \mathrm{to} \mathrm{yield} \mathrm{well-defined} \mathrm{Si} \mathrm{NCs} \mathrm{embedded}$ in a $\mathrm{SiO}_{2}$-like matrix. Hydride-terminated Si NCs were obtained from the oxide matrix via HF etching. The Si NCs were functionalized using dodecylamine and ammonium bromide to obtain nitrogen-bonded colloidal Si NCs. Details of the functionalization of Si NCs is presented in the (ESI $\dagger$ ).

\subsection{Characterization}

Fourier transformation infrared spectroscopy (FT-IR) was performed using a Nicolet Magna 750 IR spectrometer. Raman spectra were recorded on Renishaw inVia Raman Microscope. Transmission electron microscopy (TEM) and selected area electron diffraction (SAED) analyses were performed using a JOEL-2010 ( $\mathrm{LaB}_{6}$ filament) with an accelerating voltage of $200 \mathrm{keV}$. TEM samples were prepared by drop coating the Si NC suspension onto a carbon coated copper grid with a $400 \mu \mathrm{m}$ diameter hole. The NC size was averaged over 200 particles determined using ImageJ software (version 1.45).

\subsection{Time-integrated and time-resolved PL spectroscopies}

TRPL spectroscopy measurements on sub-nanosecond (sub-ns), nanosecond (ns), and microsecond ( $\mu \mathrm{s})$ time scales were carried out at room temperature on functionalized Si NCs dissolved in toluene. Si NC solutions were placed in $10 \mathrm{~mm}$ quartz cuvettes for PL measurements. For nanosecond-TRPL (referred to here as ns-TRPL) measurements, samples were excited using $400 \mathrm{~nm}$, 70 fs excitation pulses from the second harmonic output of an ultrafast amplified Ti:sapphire laser source (Coherent RegA) with a repetition rate of $250 \mathrm{kHz}$. The PL was detected using an avalanche photodiode coupled to a monochromator, and analyzed using time-correlated single photon counting unit (PicoQuant, PicoHarp 300) with a temporal resolution of $50 \pm 4$ ps. The excitation spot size was $\sim 20 \mu \mathrm{m}$ at the center of the solution, and the average excitation fluence was varied from 0.02 to $2.94 \mathrm{~mJ} \mathrm{~cm}^{-2}$. The time-integrated PL (TIPL) spectra were detected by a thermoelectrically-cooled CCD coupled to a monochromator. For comparison, steady-state PL (SSPL) measurements were also carried out using a continuouswave $406 \mathrm{~nm}$ laser diode with average excitation power of $4.8 \mathrm{~mW}$. All the PL spectra were corrected to the spectral response of the system.

Microsecond-TRPL (referred to here as $\mu \mathrm{s}$-TRPL) was performed using $400 \mathrm{~nm}, 50$ fs excitation pulses from a frequencydoubled amplified Ti:sapphire laser source (Coherent Legend Elite) operating at a repetition rate of $1 \mathrm{kHz}$. The average excitation power was $4.5 \mathrm{~mW}$ focused to a spot size of $\sim 500 \mu \mathrm{m}$, resulting in a fluence of $2.29 \mathrm{~mJ} \mathrm{~cm}^{-2}$. The PL was collected by a fast silicon photodiode and amplifier (rise time of $\sim 45 \mathrm{~ns}$, fall time $<\mu \mathrm{s}$ ) connected to a $300 \mathrm{MHz}$ oscilloscope. Bandpass filters with $10 \mathrm{~nm}$ bandwidth were used to select emission wavelengths between $450 \mathrm{~nm}$ and $760 \mathrm{~nm}$. A $435 \mathrm{~nm}$ longpass filter was used in all the PL measurements to block scattered excitation light. The transmission of the longpass filter was $86 \%$ at $450 \mathrm{~nm}$, followed by a sharp decrease in transmission at shorter wavelengths, as shown in Fig. S1 (ESI $\dagger$ ). The filter cut-off region is shown as a blue shaded area in all the PL spectral plots.

\section{Results and discussion}

The schemes for ammonia and dodecylamine functionalized $\mathrm{Si}$ NCs are shown in Fig. 1(a) and (c), respectively. Both samples exhibit visible blue PL when excited by $365 \mathrm{~nm}$ UV lamp, as shown in Fig. 1(b) and (d). Selected-area electron diffraction (Fig. S2, ESI $\dagger$ ) shows high crystallinity for the ammonia and dodecylamine functionalized Si NCs. Typical TEM images of the functionalized Si NCs are shown in Fig. 1(e), (f) and Fig. S3(a) and (c) (ESI $\dagger$ ). Two different sizes of dodecylamine and ammonia functionalized Si NCs were prepared to determine the effect of NC size on the PL emission dynamics for this particular set of functionalized Si NCs. Analysis of TEM micrographs shows that ammonia functionalized Si NCs have average diameters of $3.4 \pm 0.4 \mathrm{~nm}$ and $5.7 \pm 0.6 \mathrm{~nm}$ (Fig. 1 (g) and $\mathrm{S} 3$ (b), $\mathrm{ESI} \dagger$ ) while dodecylamine functionalized Si NCs have average diameters of $3.1 \pm 0.4 \mathrm{~nm}$ and $5.8 \pm 0.7 \mathrm{~nm}$ average diameters (Fig. 1(h) and Fig. S2(d), ESI $\dagger$ ).

Surface passivation was verified using FTIR and Raman spectroscopies, as shown in Fig. 2. FTIR measurements revealed that the functionalized Si NCs exhibit partial surface oxidation, as evidenced by a Si-O-Si stretching mode ca. $1120 \mathrm{~cm}^{-1}$. Fig. 2(a) shows that both neat ammonium bromide and ammonia functionalized Si NCs show strong $\mathrm{N}-\mathrm{H}$ absorptions at $3000-3100 \mathrm{~cm}^{-1}$, consistent with ammonia passivation of the Si surface. On the other hand, Fig. 2(b) shows a characteristic $\mathrm{N}-\mathrm{H}$ stretch at $c a .3300 \mathrm{~cm}^{-1}$ in neat dodecylamine, which is absent from the Si NC spectrum, consistent with an amine group attaching to the Si surface. Features attributable to $\mathrm{C}-\mathrm{H}$ stretches of the alkyl chain are observed at $c a .2900$ and $1470 \mathrm{~cm}^{-1}$ in neat dodecylamine and functionalized Si NCs. $\mathrm{Si}-\mathrm{N}$ vibrational modes at $c a .840 \mathrm{~cm}^{-1}$ for both ammonia functionalized Si NCs and dodecylamine functionalized $\mathrm{Si}$ NCs were observed in Raman spectra (Fig. 2(c) and (d)) further confirming the nitrogen bonding to the Si surface. Si-Si vibrational modes were also observed at $c a .520 \mathrm{~cm}^{-1}$.

Fig. 3(a) and (b) show the TIPL of the ammonia and dodecylamine functionalized Si NCs, respectively. The highenergy shoulder of the PL is truncated by the transmittance of the optical filter (Fig. S1, ESI $\dagger$ ). Interestingly, the TIPL spectra show size-independent PL spectral profiles, with peak emission at $\sim 493 \mathrm{~nm}$ for ammonia functionalized Si NCs and at $\sim 479 \mathrm{~nm}$ for dodecylamine functionalized Si NCs. Size-independent PL emission is incompatible with quantum confined core state emission. $^{22-24}$ In addition, the measured PL spectra are significantly blue shifted with respect to QC Si core bandgap transitions reported elsewhere for Si NCs with similar diameters (i.e., QC emission at $\sim 700 \mathrm{~nm}$ for $3.1 \mathrm{~nm}$ and $\sim 795 \mathrm{~nm}$ for $5.8 \mathrm{~nm} \mathrm{Si} \mathrm{NCs}) .{ }^{25,26}$ It is therefore unlikely that the observed size-independent blue PL in these functionalized Si NCs arises as a result of core state recombination. 

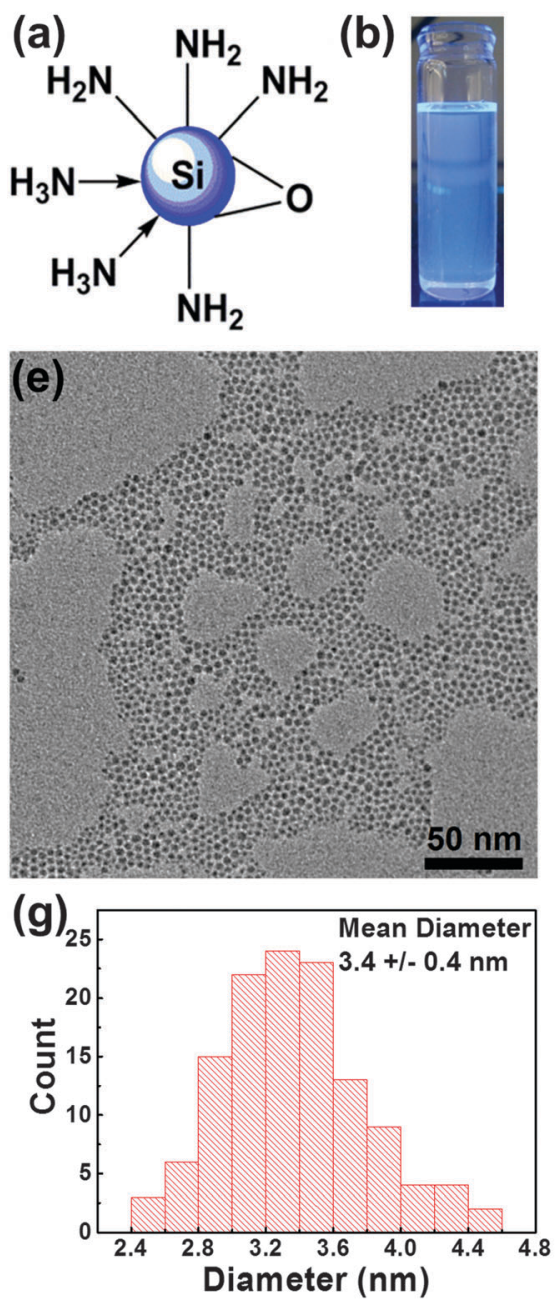

(b)
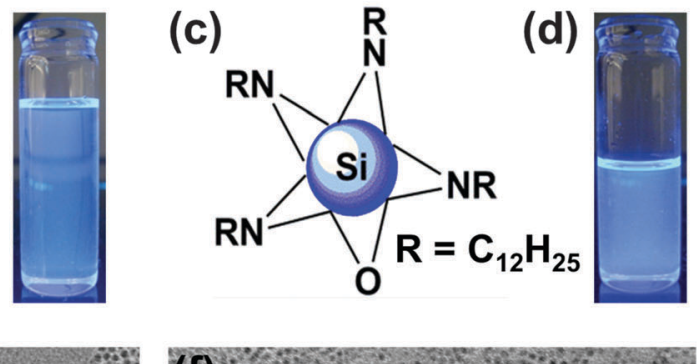

(c)

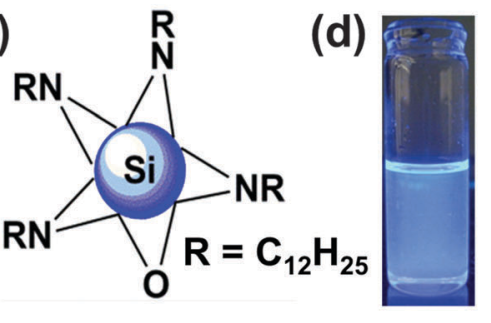

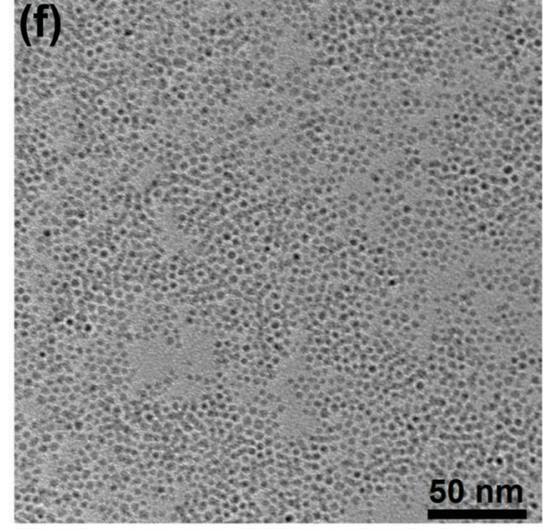

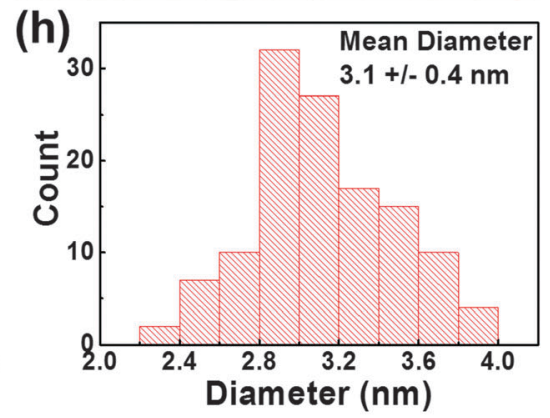

Fig. 1 Schemes for (a) ammonia and (c) dodecylamine functionalized Si NCs. Visible PL of (b) ammonia and (d) dodecylamine functionalized Si NCs excited by $365 \mathrm{~nm}$ UV lamp. TEM images of $\sim 3 \mathrm{~nm}$ (e) ammonia and (f) dodecylamine functionalized Si NCs. Histograms of the NC size distribution showing an average diameter of (g) $3.4 \pm 0.4 \mathrm{~nm}$ for ammonia functionalized Si NCs and (h) $3.1 \pm 0.4 \mathrm{~nm}$ for dodecylamine functionalized Si NCs.

Fig. 3(c) and (d) show the ns-TRPL emission dynamics for $\sim 3 \mathrm{~nm}$ and $\sim 6 \mathrm{~nm}$ ammonia and dodecylamine functionalized Si NCs, respectively, measured at $470 \mathrm{~nm}$. The PL lifetime exhibits a bi-exponential decay,

$$
I(t)=A_{1} \mathrm{e}^{\left(-t / \tau_{1}\right)}+A_{2} \mathrm{e}^{\left(-t / \tau_{2}\right)}+C,
$$

where $\tau_{1}$ and $\tau_{2}$ are the decay times and $C$ is a constant offset much smaller than $A_{1}$ and $A_{2}$. As shown in Fig. 3(c) and (d), the PL dynamics of both functionalized Si NCs at an emission wavelength of $470 \mathrm{~nm}$ is independent of nanocrystal size. As summarized in Table S1 (ESI $\dagger$ ), ammonia functionalized Si NCs have lifetimes of $\tau_{1}=1.81 \mathrm{~ns}$ and $\tau_{2}=6.12 \mathrm{~ns}$ for $d=$ $3.4 \mathrm{~nm}$ and $\tau_{1}=1.81 \mathrm{~ns}$ and $\tau_{2}=6.10 \mathrm{~ns}$ for $d=5.7 \mathrm{~nm}$. Dodecylamine functionalized Si NCs exhibit slightly shorter PL lifetimes of $\tau_{1}=1.46 \mathrm{~ns}$ and $\tau_{2}=5.76 \mathrm{~ns}$ for $d=3.1 \mathrm{~nm}$ and $\tau_{1}=1.64 \mathrm{~ns}$ and $\tau_{2}=5.86 \mathrm{~ns}$ for $d=5.8 \mathrm{~nm}$. We also explored the dependence of the PL lifetimes on emission wavelength (Table S1 and Fig. S4, ESI $\dagger$ ). All the samples exhibit very fast PL lifetimes shorter than 7 ns. In addition, the PL lifetimes of all the samples do not vary significantly with emission wavelength (the difference is $\leq 11 \%$ between $470 \mathrm{~nm}$ to $620 \mathrm{~nm}$, as seen in Table S1, ESI $\dagger$ ). Bi-exponential PL decays are typically observed as a result of energy transfer either from core/band edge states to surface states or from smaller to larger NCs. ${ }^{27-29}$ However, the PL red-shift upon energy transfer predicted by these mechanisms is not observed here. $\mu \mathrm{s}$-TRPL measurements performed from 450 to $760 \mathrm{~nm}$ did not exhibit any long-lived PL lifetime, as shown in Fig. S5 (ESI $\dagger$ ), in stark contrast with QC emission of NCs of similar size $(3-6 \mathrm{~nm})$ that is known to exhibit a $\mu \mathrm{s}-\mathrm{PL}$ decay in the range of $20-130 \mu \mathrm{s} .{ }^{30,31}$ In addition, it was recently shown that excitation wavelength-dependent PL measurements on these samples revealed a PL red-shift with excitation wavelength from $300 \mathrm{~nm}$ to $380 \mathrm{~nm}$ and a saturation in the PL peak position ( $\sim 485 \mathrm{~nm}$ for ammonia and $\sim 473 \mathrm{~nm}$ for dodecylamine functionalized Si NCs) at $390 \mathrm{~nm}$ excitation wavelength. ${ }^{10,20}$ Based on these results, we can rule out recombination from the core states as the origin of the observed size-independent blue PL since this process is highly dependent on the size of the NC. ${ }^{31-33}$ 

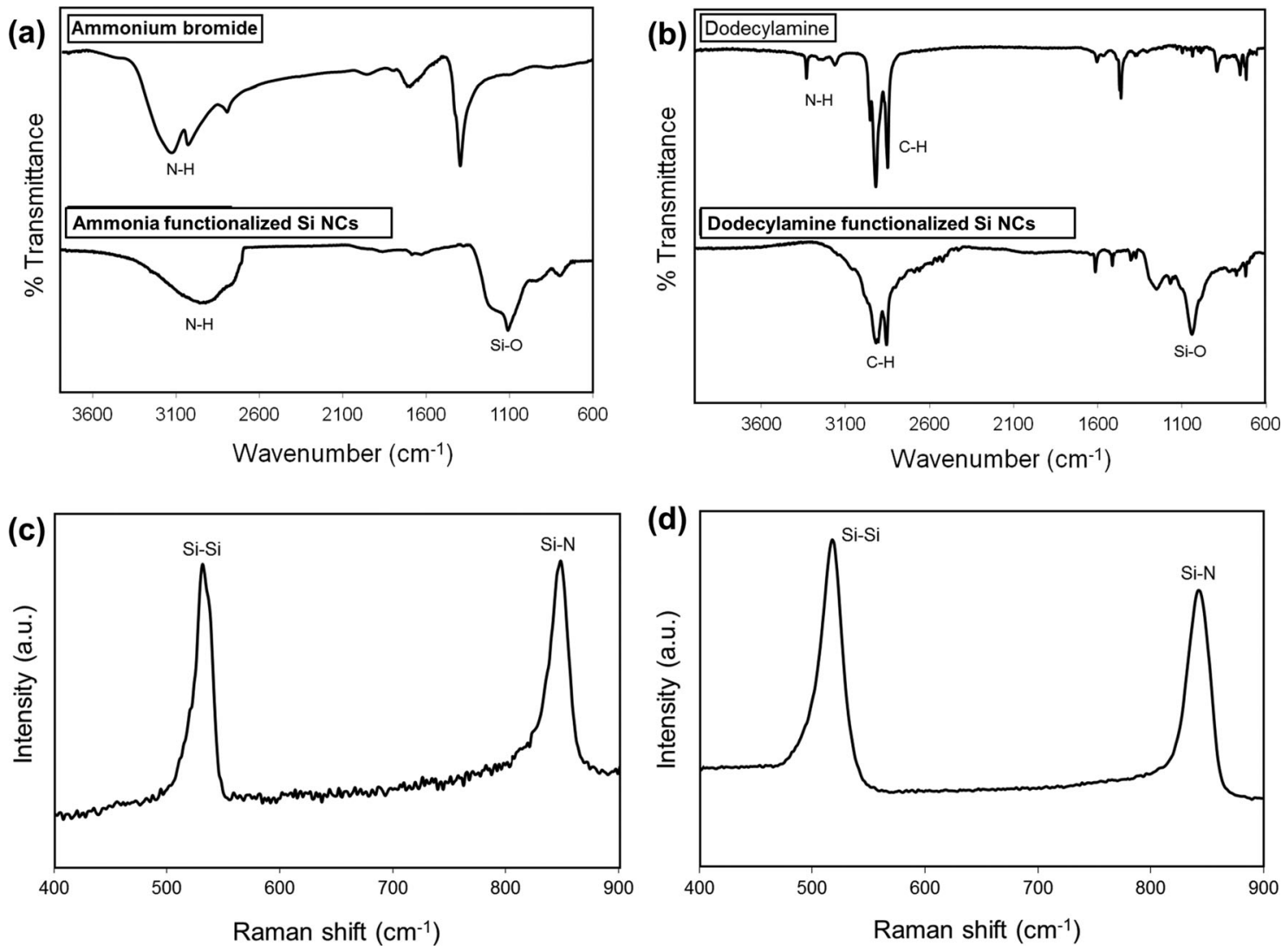

Fig. 2 FTIR spectra of (a) ammonium bromide and ammonia functionalized Si NCs and (b) dodecylamine ligand and dodecylamine functionalized Si NCs. (c and d) are the corresponding Raman spectra of functionalized Si NCs.

We estimate the radiative recombination lifetime, $\tau_{\mathrm{R}}$ using the relative PL quantum yield, QY and the weighted average lifetime, $\langle\tau\rangle$ given by ${ }^{34}$

$$
\begin{gathered}
\mathrm{QY}=\frac{\langle\tau\rangle}{\tau_{\mathrm{R}}} \\
\langle\tau\rangle=\sum_{i=1}^{n} \frac{A_{i} \tau_{i}^{2}}{A_{i} \tau_{i}}
\end{gathered}
$$

The radiative recombination rate is then given by $k_{R}=1 / \tau_{R}$. The relative $\mathrm{PL}, \mathrm{QY}$, for dodecylamine and ammonia functionalized Si NCs have recently been reported by Dasog et al. ${ }^{10,20}$ Ammonia functionalized Si NCs have a QY of $22 \%$, while dodecylamine functionalized Si NCs exhibit a QY of 32\%. Using the PL lifetimes at the peak emission wavelengths, radiative recombination rates of $k_{\mathrm{R}}=0.45 \times 10^{8} \mathrm{~s}^{-1}$ and $0.72 \times 10^{8} \mathrm{~s}^{-1}$ are obtained for ammonia and dodecylamine functionalized Si NCs, respectively. These fast radiative recombination rates suggest dipole-allowed transitions, such as observed in CdSe NCs. ${ }^{35}$ This result further confirms that the observed radiative process is unlikely due to emission from the $\mathrm{Si}$ core, since radiative recombination rates from QC states in similar size Si NCs is expected to be in the range of $10^{3}$ to $10^{5} \mathrm{~s}^{-1}$. ${ }^{11}$ The nonradiative decay rate $k_{\mathrm{NR}}$ can be computed using $\mathrm{QY}=k_{\mathrm{R}} /\left(k_{\mathrm{R}}+k_{\mathrm{NR}}\right)$. The non-radiative processes for both functionalized Si NCs are slightly faster $\left(k_{\mathrm{NR}}=1.50 \times 10^{8} \mathrm{~s}^{-1}\right.$ for dodecylamine and $k_{\mathrm{NR}}=1.59 \times 10^{8} \mathrm{~s}^{-1}$ for ammonia) than the radiative process. These efficient recombination processes lead to the observed ns-PL decay.

An extensive ns-TRPL study was conducted to further probe the nature of the blue-emitting state in this set of functionalized Si NCs (Fig. 4). By monitoring the temporal evolution of the PL spectral profile it is possible to determine the lightemitting states responsible for the observed PL since they will exhibit unique PL spectral profiles and lifetimes. ${ }^{36}$ Fig. 4(a) and (b) show the ns-TRPL intensity for the $\sim 3 \mathrm{~nm}$ ammonia and dodecylamine functionalized Si NCs, respectively, as a function of emission wavelength and time after photoexcitation. From these plots, the temporal evolution of the PL after photoexcitation was extracted, as presented in Fig. 4(c) and (d). Interestingly, the spectral profile of both functionalized Si NCs does not change with time while the peak position is consistent with the TIPL results (Fig. 3(a) and (b)). Notably, excellent agreement between the normalized temporally-resolved PL and the SSPL (Fig. 4(e) and (f)) is observed. A PL peak that is independent of time is consistent with the absence of a $\mu$ s-PL component in these Si NCs. Furthermore, the time-independent spectral profile also implies that a single emitting state is responsible for the observed ns-PL.

Based on the results of the TIPL (Fig. 3) and TRPL (Fig. 4) measurements, we conclude that the observed ns-blue PL is not from core emission. We can also rule out no-phonon hot carrier recombination described by de Boer et al. ${ }^{37}$ since the observed PL does not exhibit a PL red-shift when the size of the NCs is 

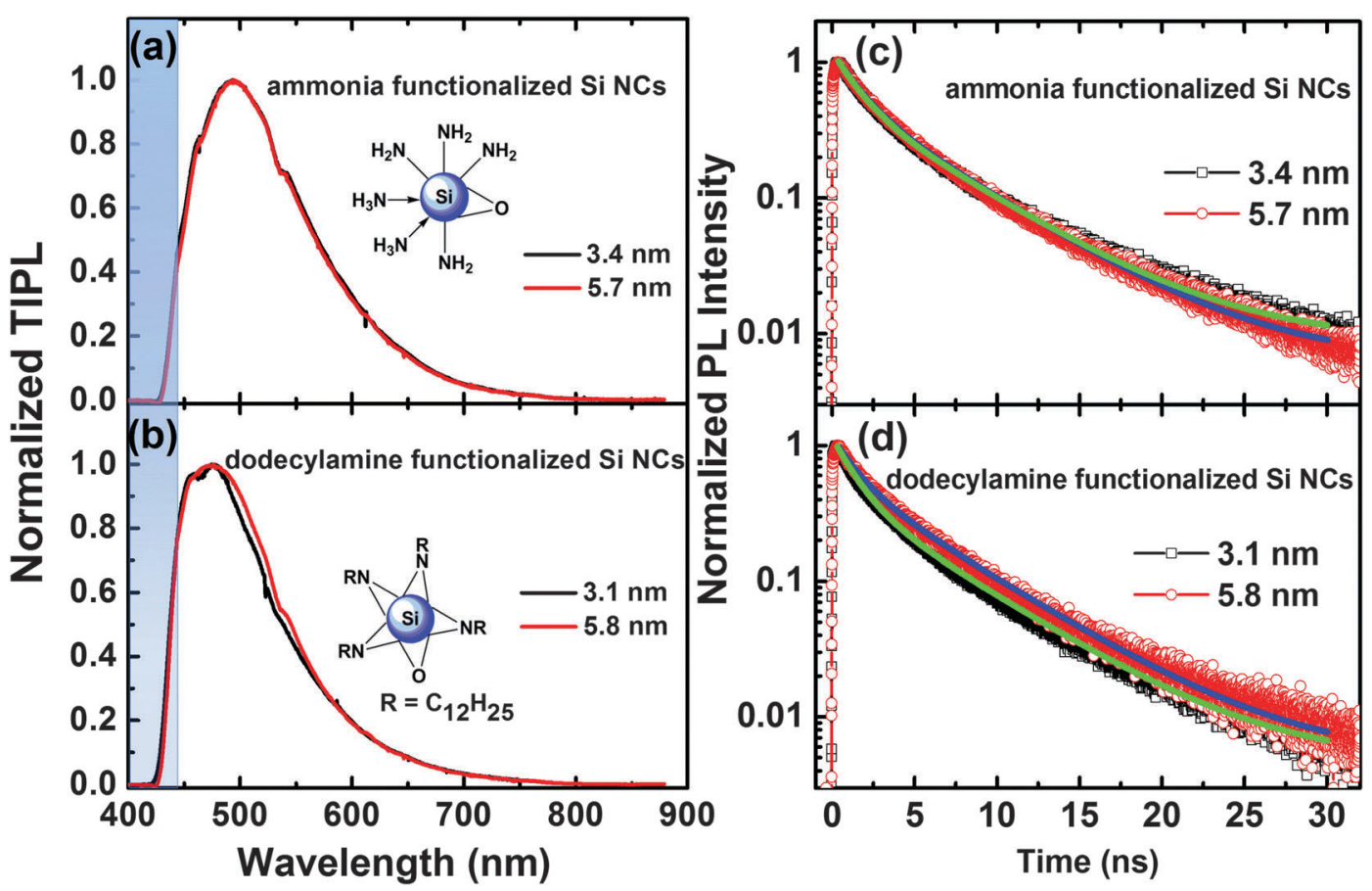

Fig. 3 TIPL of $\sim 3 \mathrm{~nm}$ (black) and $\sim 6 \mathrm{~nm}$ (red) (a) ammonia and (b) dodecylamine functionalized Si NCs excited by $400 \mathrm{~nm}$ pulses at an excitation fluence of $0.42 \mathrm{~mJ} \mathrm{~cm}^{-2}$. The shaded area represents the cut-off region of the long pass filter. The corresponding ns-TRPL decays at $470 \mathrm{~nm}$ are shown in (c) and (d), respectively. The green and blue lines are the bi-exponential fits to the $\sim 3 \mathrm{~nm}$ and $\sim 6 \mathrm{~nm}$ Si NCs, respectively. Fit parameters are given in the text and Table S1 (ESI†).

decreased, as shown in Fig. 3. It has been shown that Auger recombination could result in a PL blue-shift where carriers are promoted to higher energy levels. ${ }^{38}$ Although the time resolution of our setup prevents us from observing Auger recombination, which normally occurs on time scales $<100 \mathrm{ps}^{39}{ }^{39}$ excitationfluence-dependent PL emission and PL dynamics measurements can elucidate whether non-radiative Auger processes give rise to the observed fast blue PL. Fig. 5(a) and (b) show the excitationfluence-dependent TIPL and ns-TRPL dynamics, respectively of the $\sim 6 \mathrm{~nm}$ ammonia functionalized Si NCs. The TIPL of ammonia-functionalized Si NCs increases linearly with excitation pump fluence, inconsistent with Auger processes. The spectrally-resolved PL lifetimes are also independent of excitation fluence ( $\leq 1 \%$ variation over the fluence range used here), as shown in the inset of Fig. 5(b). In addition, the absence of PL redshift (Fig. 4(c) and (d)) at early times after photoexcitation rules out multiple carrier generation that could result in Auger recombination processes. ${ }^{40}$ Recently, Hannah et al. suggested that fast PL from Si NCs in the 400-600 nm emission bands is due to a surface layer of amorphous $\mathrm{Si}^{41}$ This is unlikely the case for our samples as we do not observe any amorphous $\mathrm{Si}-\mathrm{Si}$ peak at $\sim 480 \mathrm{~cm}^{-1}$ in our Raman spectra, ${ }^{42}$ as seen in Fig. 2(c) and (d).

Our results suggest that the observed ns-blue emission is due to radiative recombination at interface-related states by ultrafast carrier trapping or charge transfer (CT). ${ }^{34,43}$ The complete quenching of the Si core emission indicates that carrier trapping/CT depopulates the Si core at a rate much faster than intraband thermalization. Trojanek et al. showed that the carrier trapping/CT process occurs in $\sim 400 \mathrm{fs}$, approximately two orders of magnitude faster than the intraband relaxation time ( 1-10 ps) in quantum confined materials. ${ }^{44}$ Hence, we propose that the observed ns-blue PL is related to surface passivation. The FTIR and Raman spectra in Fig. 2 show both $\mathrm{Si}-\mathrm{O}$ and $\mathrm{Si}-\mathrm{N}$ surface bonds are present. However, the fast blue emission cannot be attributed to either one of these bonds. Wolkin et $a l^{45}$ and Dohnalova et $a l .{ }^{46}$ show that when the surface of Si NCs is terminated by oxygen, a PL red-shift is observed followed by an increase in the PL lifetime from ns to $\mu$ s time scales. Also, computational studies reveal that the same PL redshift was observed when oxygen-free Si NC surfaces were passivated by nitrogen. ${ }^{47}$ On the other hand, Fuzell et al. suggested that blue emission from nitrogen-bonded Si NCs is due to CT to low lying nitrogen traps while oxygen impurities generate deeper traps. ${ }^{48}$ Here, however, we associate the fast blue PL emission to silicon oxynitride $\left(\mathrm{SiO}_{x} \mathrm{~N}_{y}\right)$ species at the surface of the nanocrystals, as confirmed by XPS. ${ }^{10,20}$ Wang et al. ${ }^{49}$ proposed that both organic ligands and surface oxidation are necessary to form a novel surface state that is responsible for the ultrabright PL and that the structure of the ligand is crucial in determining whether such a state can dominate the entire process. However, it is unlikely that the emission is due to direct excitation of the $\mathrm{SiO}_{x} \mathrm{~N}_{y}$ surface group since its bandgap is between $4.5-8.0 \mathrm{eV}^{50}$ while the excitation energy used here is only $3.1 \mathrm{eV}$. Furthermore, absorption measurements shown in Fig. S6 (ESI $\dagger$ ) exhibit size-dependent absorption edges commonly observed for nanostructured $\mathrm{Si}^{30}$

The CT state, which occurs at the $\mathrm{Si} / \mathrm{SiO}_{x} \mathrm{~N}_{y}$ interface, is therefore responsible for the observed ns-blue PL, as illustrated in Fig. 6. 


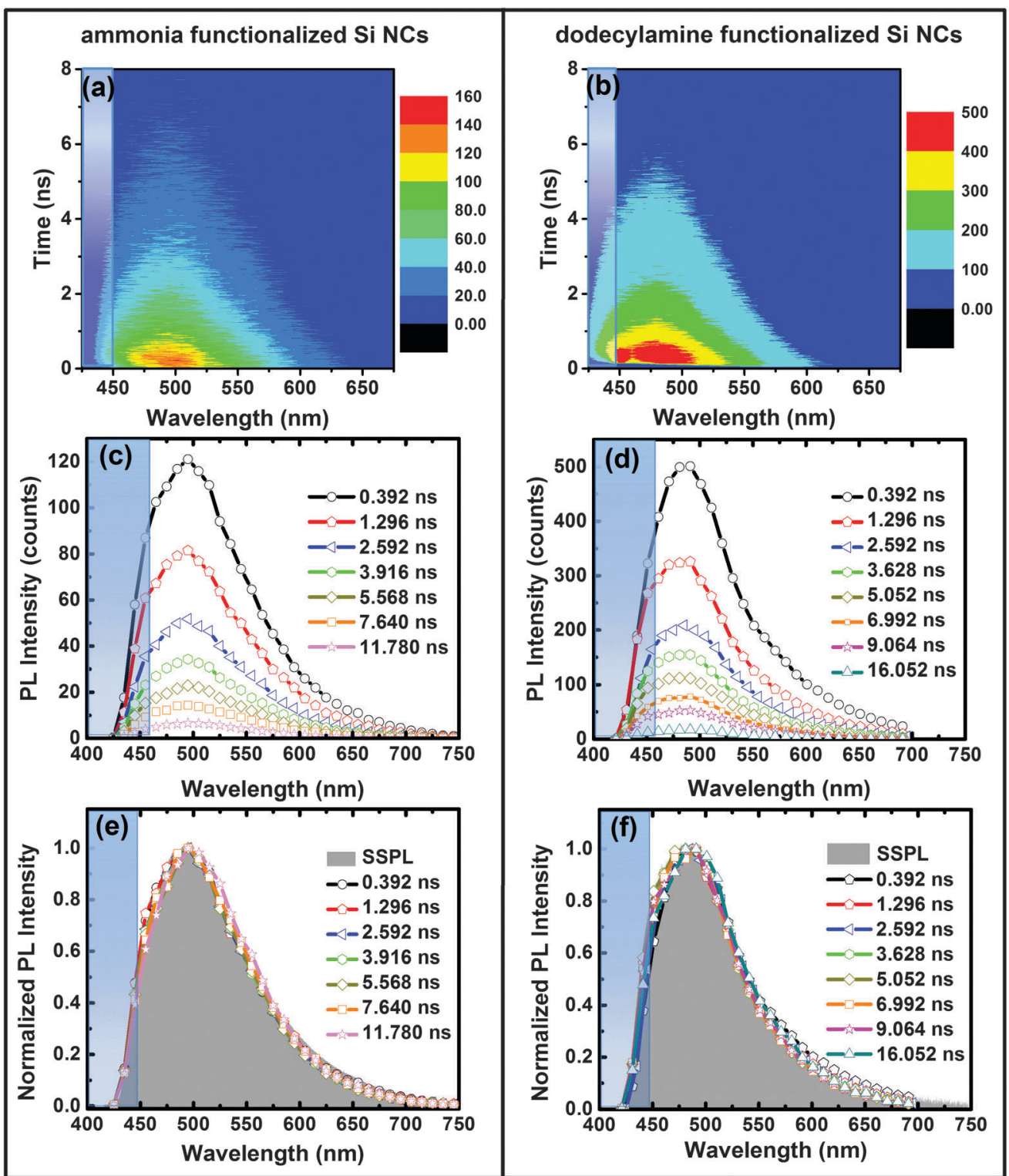

Fig. 4 TRPL intensity as a function of wavelength and time for $\sim 3 \mathrm{~nm}$ (a) ammonia and (b) dodecylamine functionalized SiNCs. The color scale represents photon counts. (c) and (d) are the corresponding temporally-resolved PL spectra at specific times after excitation and averaged within a time window of $0.4 \mathrm{~ns}$. Comparison of the SSPL (grey shaded area) and the normalized PL of (e) ammonia and (f) dodecylamine functionalized Si NCs. The blue-shaded area represents the cut-off region of the long pass filter. PL was excited by $400 \mathrm{~nm}$ pulses at an excitation fluences of $0.48 \mathrm{~mJ} \mathrm{~cm}^{-2}$ and $0.55 \mathrm{~mJ} \mathrm{~cm}^{-2}$ for ammonia and dodecylamine functionalized Si NCs, respectively.

The Si NC, with bandgap $E_{\text {gap }}^{\mathrm{QC}}(>1.1 \mathrm{eV}$ bandgap of bulk silicon), is excited by $400 \mathrm{~nm}(3.1 \mathrm{eV})$ light creating an electron-hole pair. The photoexcited electron rapidly transfers to the $\mathrm{SiO}_{x} \mathrm{~N}_{y}$ forming the CT state located at the interface. The CT thermalization process occurs at a much faster rate than the thermalization process within the bands of the Si core resulting in quenching of the QC core emission that typically emits red PL with a lifetime in the $\mu$ s time scale, as shown by the red solid downward vertical arrow in Fig. 6. After a fast thermalization within the CT state, the bound electron-hole pair recombines emitting ns-blue PL. Deibel et al. pointed out that when sufficient wavefunction overlap of the electron and hole forming the CT state occurs, significant luminescence can be achieved. ${ }^{51}$ In addition, Dasog et al. recently showed that these functionalized Si NCs exhibit solvent-polarity dependent (solvatochromic) PL, ${ }^{13,20}$ consistent with a CT state at the interface. The difference in PL spectra and lifetimes of ammonia and dodecylamine functionalized Si NCs can be attributed to the difference in structure of the $\mathrm{SiO}_{x} \mathrm{~N}_{y}$ species, which affects the CT state. Wang et al. ${ }^{49}$ pointed out that for surface-modified Si QDs a modulation in the exciton wave functions is expected due to core-ligand interactions which implies that the emission dynamics will be sensitive to the structure of the capping agents. This explains the difference in the PL quantum yield and radiative recombination rate observed in dodecylamine and ammonia functionalized Si NCs. Indeed, Augustine et al. used various amorphous silicon oxynitride 

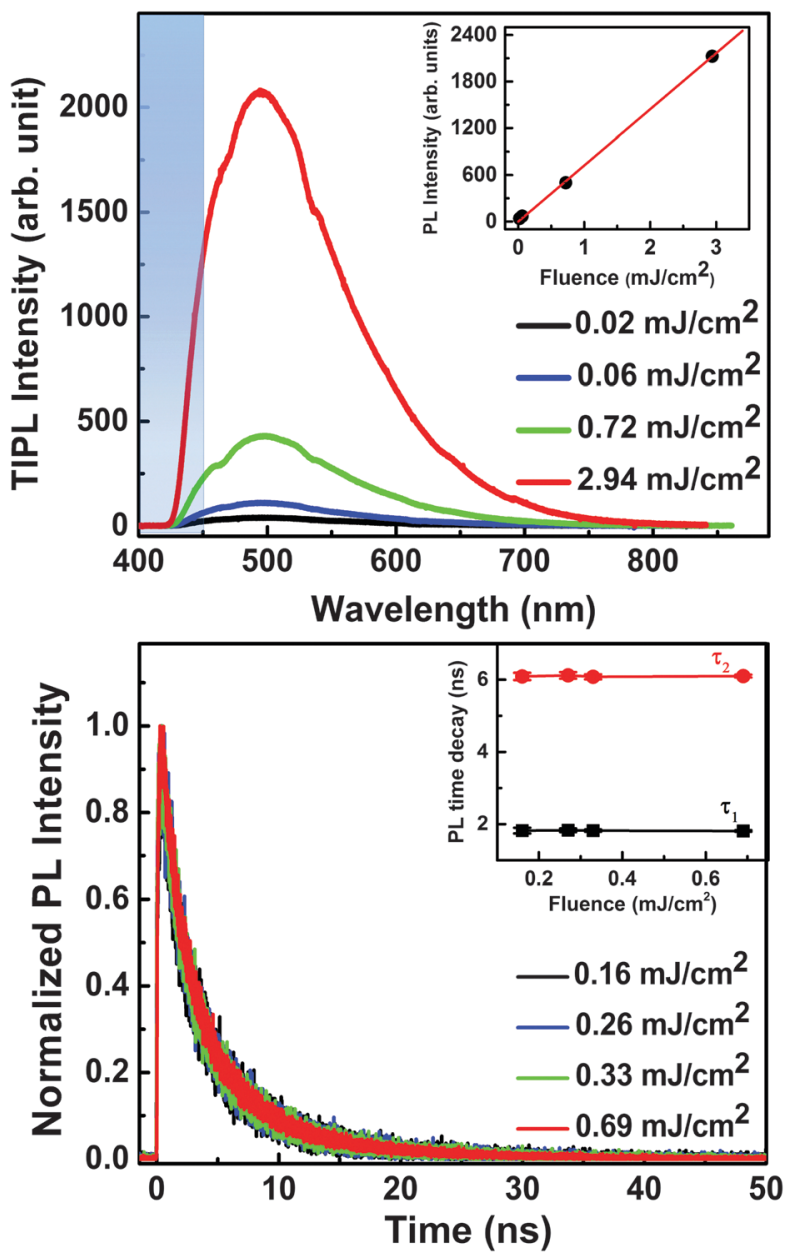

Fig. 5 Fluence-dependence of (a) TIPL and (b) ns-TRPL dynamics at $493 \mathrm{~nm}$ for $\sim 6 \mathrm{~nm}$ ammonia functionalized Si NCs. Insets show the fluence-dependence of the (a) PL intensity and (b) PL lifetimes, respectively. The blue-shaded area represents the cut-off region of the long pass filter.

$\left(\mathrm{a}-\mathrm{SiO}_{x} \mathrm{~N}_{y}: \mathrm{H}\right)$ thin films prepared by PECVD to demonstrate tunable visible PL emission with a characteristic decay of $<10 \mathrm{~ns}^{52}$ While the origin of the bi-exponential nature of the PL decay observed here is still unclear, similar bi-exponential PL emission dynamics due to CT states has been observed in polymer-fullerene systems. ${ }^{53,54}$ Loi et al. attributed the bi-exponential behavior to energy transfer followed by PL emission, ${ }^{53}$ whereas Veldman et al. suggested that bi-exponential decay dynamics could be a result of CT state distribution due to different electron-hole separations. ${ }^{54}$

The CT state emission from our functionalized Si NCs exhibits complete blue PL with no other components attributable to core emission, and is therefore a more efficient blue emitter compared to oxygen-related defects and amorphous surface layers where the blue emission is just a small part of the total PL. ${ }^{17,18}$ Compared to QC blue emission from Si cores, CT state emission is likely to exhibit higher PL QY and faster radiative recombination since it has been shown that a significant decrease in QY is observed when the Si NCs diameter is less than $2 \mathrm{~nm} .{ }^{31}$ Mastronardi et $a l .{ }^{31}$ observed that as the NC size decreases, the non-radiative recombination rate dominates the radiative rate which results in a decrease in absolute QY. Hence, blue emission from CT

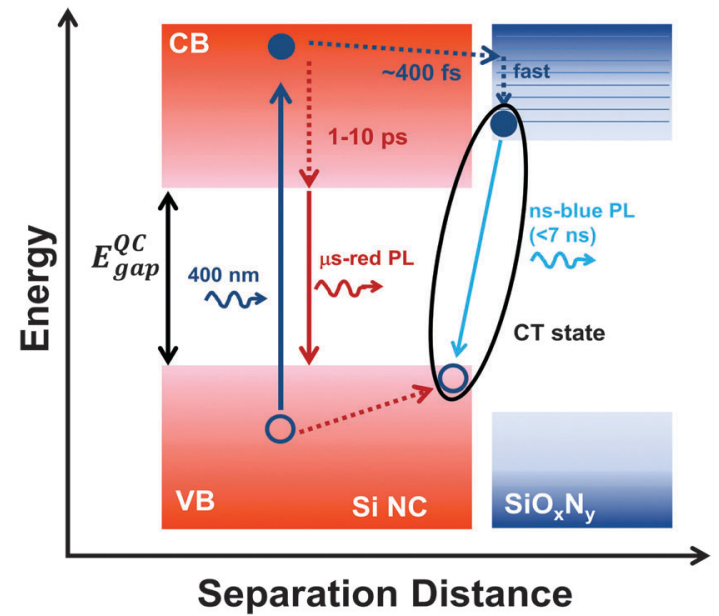

Fig. 6 Energy diagram for the CT state emission dynamics. Electrons are first photoexcited within the Si NC core and then rapidly transfer to the $\mathrm{SiO}_{x} \mathrm{~N}_{y}$, creating a CT state at the interface. The CT process quenches the emission from the Si core, which is normally expected to emit red PL over $\mu$ s time scales, as shown by the solid red downward arrow. Radiative recombination of the electron and hole in the CT state results in blue PL over ns time scales, as indicated by a solid blue downward arrow. ( $C B=$ conduction band; $V B=$ valence band). The electron transfer (dashed blue arrow) and thermalization times (dashed red downward arrow) are adopted from ref. 43.

states is a promising candidate for applications in Si-based LEDs, as luminescent tags in imaging, and in other applications where efficient emission in the blue region of spectrum is required. Understanding the CT state emission dynamics is also important for Si NC-based photovoltaics, where CT states are the intermediate process necessary for charge dissociation that can results in the generation of photocurrent. ${ }^{50}$ Since recombination from a CT state is considered an energy loss, preventing it to happen by ensuring that charge separation occurs before recombination will result in better performance of solar cells. The key to achieving this is by knowing the characteristic time of the CT state emission.

\section{Conclusions}

In summary, the PL dynamics of blue-emitting ammonia and dodecylamine functionalized Si NCs with average diameters of $\sim 3$ and $\sim 6 \mathrm{~nm}$ was investigated. The NC-size-independent blue emission is attributed to CT states at the $\mathrm{Si} / \mathrm{SiO}_{x} \mathrm{~N}_{y}$ interface with a radiative recombination rate of $\sim 5 \times 10^{7} \mathrm{~s}^{-1}$. In addition, the CT state emission spectral profile is independent of time after excitation. The size-independent PL and the absence of $\mu \mathrm{s}-\mathrm{PL}$ from all of our samples suggest a very efficient electron transfer from the Si NC core excited states to the CT state. Understanding the CT state emission dynamics in blue-emitting Si-NCs will impact their application in optoelectronic devices.

\section{Acknowledgements}

The authors acknowledge funding from the Natural Sciences and Engineering Research Council of Canada (NSERC), Canada Foundation for Innovation (CFI), Alberta Science and Research 
Investment Program (ASRIP), Alberta Innovates Technology Futures (AITF), iCiNano (iCORE Centre for Interdisciplinary Nanoscience), and University of Alberta Department of Chemistry. G. De los Reyes would like to thank AITF for support. Killam trusts, NSERC, and AITF are acknowledged by M. Dasog. We would like to thank W. C. Moffat and B. Mason for assistance with FTIR spectroscopy, and G. Popowich and D. Mullin for technical support. G. Kaufmann is thanked for assistance with Raman spectroscopy.

\section{References}

1 K. D. Hirschman, L. Tsybeskov, S. P. Duttagupta and P. M. Fauchet, Nature, 1996, 384, 338-341.

2 K. Y. Cheng, R. Anthony, U. R. Kortshagen and R. J. Holmes, Nano Lett., 2010, 10, 1154-1157.

3 G. Conibeer, M. Green, R. Corkish, Y. Cho, E. C. Cho, C. W. Jiang, T. Fangsuwannarak, E. Pink, Y. Huang and T. Puzzer, et al., Thin Solid Films, 2006, 511, 654-662.

4 F. Maier-Flaig, J. Rinck, M. Stephan, T. Bocksrocker, M. Bruns, C. Kübel, A. K. Powell, G. A. Ozin and U. Lemmer, Nano Lett., 2013, 13, 475-480.

5 F. Erogbogbo, K.-T. Yong, I. Roy, G. Xu, P. N. Prasad and M. T. Swihart, ACS Nano, 2008, 2, 873-878.

6 S. Pradhan, S. Chen, J. Zou and S. M. Kauzlarich, J. Phys. Chem. C, 2008, 112, 13292-13298.

7 Y. Kanemitsu, J. Lumin., 2002, 100, 209-217.

8 E. G. Barbagiovanni, D. J. Lockwood, P. J. Simpson and L. V. Goncharova, Appl. Phys. Rev., 2014, 1, 011302.

9 G. Ledoux, J. Gong, F. Huisken, O. Guillois and C. Reynaud, Appl. Phys. Lett., 2002, 80, 4834.

10 M. Dasog, G. B. De los Reyes, L. V. Titova, F. A. Hegmann and J. G. C. Veinot, ACS Nano, 2014, 8(9), 9636.

11 K. Dohnalová, T. Gregorkiewicz and K. Kůsová, J. Phys.: Condens. Matter, 2014, 26, 173201.

12 M. L. Mastronardi, F. Maier-Flaig, D. Faulkner, E. J. Henderson, C. Kübel, U. Lemmer and G. A. Ozin, Nano Lett., 2011, 12, 337.

13 B. Goller, S. Polisski, H. Wiggers and D. Kovalev, Appl. Phys. Lett., 2010, 97, 041110.

14 D. S. English, L. E. Pell, Z. Yu, P. F. Barbara and B. A. Korgel, Nano Lett., 2002, 2, 681.

15 J. Valenta, A. Fucikova, I. Pelant, K. Kůsová, K. Dohnalová, A. Aleknavičius, O. Cibulka, A. Fojtík and G. Kada, New J. Phys., 2008, 10, 073022.

16 R. M. Sankaran, D. Holunga, R. C. Flagan and K. P. Giapis, Nano Lett., 2005, 5, 537.

17 E. Lioudakis, A. Othonos and A. G. Nassiopoulou, Appl. Phys. Lett., 2007, 90, 171103.

18 D. C. Hannah, J. Yang, N. J. Kramer, G. C. Schatz, U. R. Kortshagen and R. D. Schaller, ACS Photonics, 2014, 1, 960.

19 S. Yang, W. Li, B. Cao, H. Zeng and W. Cai, J. Phys. Chem. C, 2011, 115, 21056-21062.

20 M. Dasog, Z. Yang, S. Regli, T. M. Atkins, A. Faramus, M. P. Singh, E. Muthuswamy, S. M. Kauzlarich, R. D. Tilley and J. G. C. Veinot, ACS Nano, 2013, 7, 2676-2685.
21 M. Dasog and J. C. G. Veinot, Phys. Status Solidi A, 2012, 209(10), 1844-1846.

22 G. Ledoux, J. Gong, F. Huisken, O. Guillois and C. G. Reynaud, Appl. Phys. Lett., 2002, 80, 4834.

23 C. Delerue, G. Allan and M. Lannoo, Phys. Rev. B, 1993, 48, 11024.

24 V. A. Belyakov, V. A. Durdov, R. Lockwood and A. Meldrum, Adv. Opt. Technol., 2008, 2008, 279502.

25 A. Sa'ar, Y. Reichman, M. Dovrat, D. Krapf, J. Jedrzejewski and I. Balberg, Nano Lett., 2005, 5, 2443.

26 O. Wolf, M. Dasog, Z. Yang, I. Balberg, J. G. C. Veinot and O. Millo, Nano Lett., 2013, 13, 2516.

27 J. Foreman, J. Li, H. Peng, S. Choi, H. Everitt and J. Liu, Nano Lett., 2006, 6, 1126-1130.

28 O. A. Ajayi, N. C. Anderson, M. Cotlet, N. Petrone, T. Gu, A. Wolcott, F. J. Hone, J. S. Owen and C. W. Wong, Appl. Phys. Lett., 2014, 104, 17101-17105.

29 C. R. Kagan, C. B. Murray, M. Nirmal and M. G. Bawendi, Phys. Rev. Lett., 1996, 76, 1517-1520.

30 C. Garcia, B. Garrido, P. Pellegrino, R. Ferrer, J. A. Moreno, J. R. Morante, L. Pavesi and M. Cazzanelli, Appl. Phys. Lett., 2003, 82, 1595.

31 M. L. Mastronardi, F. Maier-Flaig, D. Faulkner, E. J. Henderson, C. Kübel, U. Lemmer and G. A. Ozin, Nano Lett., 2011, $12,337$.

32 Z. Yang, G. B. De los Reyes, L. V. Titova, I. Sychugov, M. Dasog, J. Linnros, F. A. Hegmann and J. G. C. Veinot, ACS Photonics, 2015, 2(5), 595-605.

33 T.-Y. Kim, N.-M. Park, K.-H. Kim, G. Y. Sung, Y.-W. Ok, T.-Y. Seong and C.-J. Choi, Appl. Phys. Lett., 2004, 85, 5355.

34 D. S. English, L. E. Pell, Z. Yu, P. F. Barbara and B. A. Korgel, Nano Lett., 2002, 2(7), 681.

35 C. de Mello Donega, S. G. Hickey, S. F. Wuister, D. Vanmaekelbergh and A. Meijerink, J. Phys. Chem. B, 2003, 107, 489-496.

36 M. Sykora, L. Mangolini, R. Schaller, U. Kortshagen, D. Jurbergs and V. Klimov, Phys. Rev. Lett., 2008, 100, 067401.

37 W. D. A. M. de Boer, D. Timmerman, K. Dohnalova, I. N. Yassievich, H. Zhang, W. J. Buma and T. Gregorkiewicz, Nat. Nanotechnol., 2010, 5, 878.

38 D. Diamare, M. Wojdak, S. Lettieri and A. J. Kenyon, J. Lumin., 2013, 136, 57-62.

39 C. Sevik and C. Bulutay, Phys. Rev. B: Condens. Matter Mater. Phys., 2008, 77, 125414.

40 R. D. Schaller, M. Sykora, S. Jeong and V. I. Klimov, J. Phys. Chem. B, 2006, 110, 25332-25338.

41 D. C. Hannah, J. Ynag, N. J. Kramer, G. C. Schatz, U. R. Kortshagen and R. D. Schaller, ACS Photonics, 2014, 1, 960.

42 X. L. Wu, G. G. Siu, S. Tong, X. N. Liu, F. Yan, S. S. Jiang, X. K. Zhang and D. Feng, Appl. Phys. Lett., 1996, 69, 523.

43 L. Tsybeskov, J. V. Vandeshev and P. M. Fauchet, Phys. Rev. B: Condens. Matter Mater. Phys., 1994, 49, 7821-7824.

44 F. Trojanek, K. Neudert, P. Maly, K. Dohnalova and I. Pelant, J. Appl. Phys., 2006, 99, 116108. 
45 M. V. Wolkin, J. Jorne, P. M. Fauchet, G. Allan and C. Delerue, Phys. Rev. Lett., 1999, 82, 197.

46 K. Dohnalova, A. N. Poddubny, A. A. Prokofiev, W. D. A. M. de Boer, C. P. Umesh, J. M. J. Paulusse, H. Zuilhof and T. Gregorkiewicz, Light: Sci. Appl., 2013, 2, e47.

47 X. Pi, X. Chen, Y. Ma and D. Yang, Nanoscale, 2011, 3, 4584. 48 J. Fuzell, A. Thibert, T. M. Atkins, M. Dasog, E. Busby, J. G. C. Veinot, S. M. Kauzlarich and D. S. J. Larsen, J. Phys. Chem. Lett., 2013, 4, 3806.

49 L. Wang, Q. Li, H.-Y. Wang, J.-C. Huang, R. Zhang, Q.-d. Chen, H.-L. Xu, W. Han, Z.-Z. Shao and H.-B. Sun, Light: Sci. Appl., 2014, 4, e245.
50 V. A. Gritsenko, Silicon Nitride in Electronic, Elsevier, New York, 1998, pp. 138-187.

51 C. Deibel, T. Strobel and V. Dyakonov, Adv. Mater., 2010, 22, 4097-4111.

52 B. H. Augustine, E. A. Irene, Y. J. He, K. J. Price, L. E. McNeil, K. N. Christensen and D. M. Maher, J. Appl. Phys., 1995, 78, 4020.

53 M. A. Loi, S. Toffanin, M. Muccini, M. Forster, U. Scherf and M. Scharber, Adv. Funct. Mater., 2007, 17, 2111.

54 D. Veldman, Ö. Ipek, S. C. J. Meskers, J. Sweelssen, M. M. Koetse, S. C. Veenstra, J. M. Kron, S. S. van Bavel, J. Loos and R. A. Janssen, J. Am. Chem. Soc., 2008, 130, 7721. 